\title{
Design Principles for a Unified Statistical Data/Metadata System
}

\author{
Daniel W. Gillman \\ Martin V. Appel \\ William P. LaPlant, Jr. \\ U.S. Bureau of the Census \\ Washington, DC 20233
}

\begin{abstract}
This paper discusses a design proposal for a unified statistical data and metadata system at the U.S. Bureau of the Census and the principles guiding that design. Many statistical agencies have begun to disseminate their data via the internet. All the major program areas within the Bureau of the Census have begun internet data dissemination projects. If each data dissemination system is developed and implemented independently, then the statistical agency runs the risk that people seeking data will need to learn several different systems. Worse, it might be difficult to find and combine data from different sources because of incompatibilities among the systems. Through cooperation, the use of metadata standards, and the use of common (internet) tools, it should be possible to provide a common "look and feel" and seamless access to all agency data: a unified statistical data/metadata system. The paper will describe the principles used to guide the design, necessary standards, software tools, and architecture of the proposed system.
\end{abstract}

\section{Introduction}

The U.S. Bureau of the Census (BOC) has begun to disseminate some of its data on the internet. Each major program area in the agency has started a project to make its data available that way. Rich metadata repositories, fully integrated with the data, are necessary for users to understand the data they are seeking and to answer common questions about the data. We will call these end-user data dissemination systems statistical information systems (SIS).

The $\mathrm{BOC}$ is a stovepipe organization. Each group responsible for a particular set of surveys works independently of the others. This has resulted in much duplication of effort and a lack of coordinated, cohesive projects over time.

Studies have shown that most users of BOC data are not looking for data from a particular source. They want a data set because it contains the variables or geographic coverage they need. They do not care to know the particular survey used to collect the data or to know how the BOC is organized.

SIS's which are designed independently will not have many features in common. Some common problems may be:
- lack of a common user interface;

- poor interconnectivity between systems;

- lack of consistent functions, or similar functions which work in inconsistent ways;

- inability to link data sets from different sources;

- lack of common terminology or ability to link common concepts.

So, there is a need to coordinate among the SIS development efforts under way. One impractical solution would be to combine resources and build one large SIS for the agency. This will not work for many reasons. Alternatively, through coordination, the use of metadata standards, and the use of common software tools it should be possible to provide a common "look and feel" and seamless access to all agency data and metadata: a unified statistical data/metadata system. This paper will describe the principles used to guide the design, some important metadata standards, useful software tools, and a proposed architecture for a unified statistical data/metadata system at the BOC.

\section{Statistical information systems}

Here we define, describe the functions, and outline an architecture for a typical SIS.

\subsection{Definitions}

Statistical metadata is descriptive information or documentation about statistical data, i.e. microdata or macrodata. Statistical metadata facilitates sharing, querying, and understanding of statistical data over the lifetime of the data.

The types of statistical data are described as follows: - Microdata - data on the characteristics of units of a population, such as individuals, households or establishments, collected by a census, survey, or experiment;

- Macrodata - data derived from microdata by statistics on groups or aggregates, such as counts, means, or frequencies.

The extensive nature of statistical metadata lends itself to categorization into three components or levels: - Systems - the information about the physical characteristics of the application's data sets, such as location, record layout, database schema, media, size, etc;

- Applications - the descriptive information about the 
application's products and processes, such as sample designs, questionnaires, software, variable definitions, edit specifications, etc;

- Administrative - the management information, such as budgets, costs, schedules, etc.

The systems, applications, and administrative components help to differentiate the sources and uses of statistical metadata.

A statistical information system is an on-line system which allows for the following:

- statistical data access and retrieval;

- statistical metadata queries;

- mathematical and graphical summarization of data;

- navigation across concepts for locating information;

- graphical user interface driven access;

- access to survey design documentation.

\subsection{Features and organization}

The main features of a SIS are:

- statistical data database;

- metadata repository;

- survey documentation document library;

- user interface;

- data manipulation and analysis tool set;

- metadata/documentation creation and update tools;

- feature interconnection infrastructure;

- interface for access with other information systems;

- security matrix to protect confidential information.

The statistical data database holds the microdata and macrodata products of the survey(s) within the scope of the SIS. This database will probably be distributed, relational, support $\mathrm{SQL}$, and be optimized for performing queries. A data warehouse, which is designed for optimizing queries, could be implemented here. However, efficient and effective data warehouse implementations require standardized data elements; an issue statistical agencies are only beginning to address.

The metadata repository and document library provide the user the information to understand the data. A complete and effective metadata repository will be based on a metamodel describing parts of the underlying survey designs, processing, analyses, and data sets. Agency wide metadata standards are an effective way to ensure all the information and their relationships are available for the user.

The user interface, data manipulation and analysis tools, and metadata/documentation creation and update tools provide the user with the access to the data, metadata, and documentation. Many tools will have to be provided to ensure all functionality is covered. Examples include:

- SQL and reporting tools for database query;

- SAS and other statistical packages for data manipulation and analyses;

- Word processors and templates for creating and updating documents;

- Navigation tools (e.g. graphical user interfaces) for users to gain access to the information system;

- Internet tools.
An effective SIS will be driven by the metadata and provide context sensitive help. If a user needs the definition for a variable, it should be available at the click of a button. The metadata and documentation which describes a survey and its data needs to be searchable for users to locate the data they need. Finally, key word and hyper-text searches are effective ways for locating documents in the document library.

The feature interconnection infrastructure is the underlying network of connections and interfaces which make the information system work. The user interface must be able to call the tools which access data, metadata, or documentation. Queries against the database or metadata repository must be fast and accurate for the system to work properly.

In order for a SIS to work with other SIS's in a statistical agency, there must be a mechanism for interconnecting the systems. There are many possible architectures for achieving this interconnectivity, but minimizing the number of interfaces is optimal. This will be discussed further in chapter 3 , but it is important to recognize that interconnectivity must be part of the design; it does not come for free.

The security matrix concept is important for providing access to many kinds of users while keeping some data or other information confidential. Some SIS's will be designed for both agency survey designers and analysts and for outside users. Some microdata cannot be released to the public, but still must be made available to the designers and analysts for a particular survey. In addition, at the BOC, confidentiality suppression algorithms and congressional apportionment algorithms are metadata which need limited access.

Figure 1 below shows a scheme for how these concepts might fit together to form a SIS.

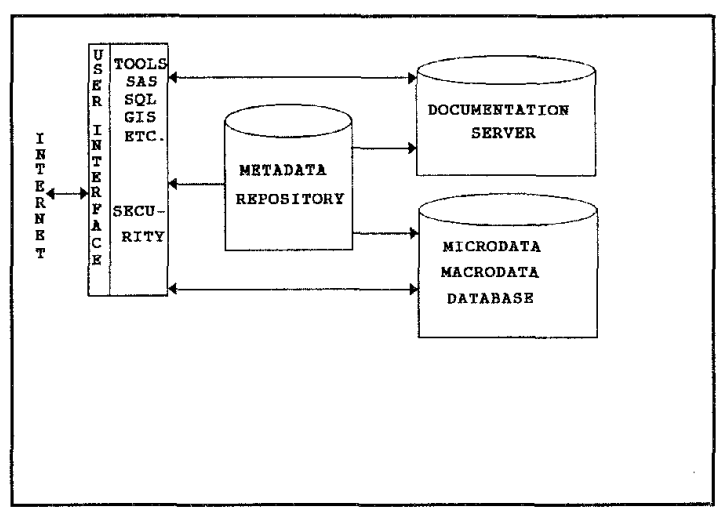

Figure 1. SIS Schema

\subsection{Limitations}

The details presented above for the design of a SIS have some limitations. The most important is the scope of the information system, i.e. for how many survey 
programs is data available in a particular SIS. The way the BOC is going about its development, there will be at least one SIS for each major program area. Thus, the problem of unifying the SIS's must be solved.

Currently, there is no agreed upon set of metadata which will be common to all the SIS's under development at the BOC. With no agreed upon set of metadata, the metamodels used to build the metadata repositories for each SIS will be different, too. These differences will make it difficult for users to search for data across SIS's. A related problem is that data element names and definitions are not standard across survey programs. It will be much harder for users to link data sets across SIS's without common or standard data definitions.

Problems may also occur with the databases and repositories in this design. Large numbers of users requesting data or metadata at the same time will put a strain on even the best designed systems.

As we shall see later, a unified system can be greater than the sum of its parts. The models describing the data and metadata for each individual SIS are designed for each of those systems and may not adequately address the information potential of the agency as a whole. A unifying model may be necessary to ensure information access across distributed SIS's.

Most of these limitations will be addressed in the following sections.

\section{Principles of design}

Here the principles on which a unified data/metadata system should be designed are described.

\subsection{Internet software}

With the advent of cheap, fast, and large data storage capabilities, electronic access to statistical data has become a reality. Now that the internet, especially the World Wide Web (WWW), is gaining wide use, creating systems with WWW access makes excellent business sense. More people all the time are gaining access and using the internet. So, there is an evergrowing group of people who know how to use WWW software.

The growing familiarity and ease of access to the WWW make it an easy choice for the development of a user interface to a SIS. Commonly used WWW browsers, such as Netscape and Mosaic, are familiar to most users. Users know how to navigate the Web with these browsers.

The internet allows anyone with a connection access to systems which are on it. So, the developer of a SIS need not worry about providing LAN or WAN connections to remote users. There will always be some who want access to a SIS who do not have an internet connection, but the number of people and organizations is decreasing all the time.

The internet provides or uses several mechanisms that make it easy (but sometimes slow) to access information. Hyper-Text Markup Language (HTML), hyper-text search capabilities, and Wide Area Information Search (WAIS) make document or information searching much easier. There is no need to use proprietary software when the tools are available on the internet.

Common Gateway Interface (CGI) provides an Application Program Interface (API) for WWW applications, making it possible to read the results of running a program on a Web page.

\subsection{Standard or common software}

Using software which adheres to national or international standards increases flexibility in developing a SIS and facilitates its use. Users can be expected to have seen or be familiar with common or standardized software. Two familiar examples are SQL and SAS.

SQL is maintained as an international standard and is undergoing new development to meet the needs of multimedia and object oriented applications. One should not expect that users of a SIS can write SQL queries, but it should be incorporated into the design of a SIS along with any relational database application.

The $\mathrm{BOC}$ has recently signed a broad licensing agreement with SAS and will install the software agency wide. SAS software includes all the popular statistical analysis tests and functions. Since it is used so widely, a SIS developer needs to make it available for users to perform their own data analyses

IRDS (Information Resource Dictionary System) is a standard for organizing and maintaining metadata. It has not received wide use; currently one software vendor sells an ANSI compliant system. IRDS suffers in part because of competing standards. There are two versions of IRDS (ISO and ANSI) which are not completely compatible, and PCTE (Portable Common Tool Environment) is a software engineering version of the same idea. However, PCTE has no vendor implementations either.

As a metadata repository development tool, an IRDS system is ideal because of great flexibility. The modeling capability can handle changes much more easily than a typical relational database management system. The modeling scheme implements EntityRelationship-Attribute schemas and does not address objects. Still, it may be very useful for implementing metadata repositories.

\subsection{Metadata standards}

Fundamental to the design of a SIS is the metadata component. The metadata are necessary for users to be able to locate, understand, and use statistical data. Well designed and complete metadata repositories provide efficient and effective access to the metadata, and it is through standards that the repositories can be measured for completeness. Standards also promote communication between systems through the assurance 
of shared and sharable information.

The BOC is currently developing a standard for statistical metadata: the Standard for Survey Design and Statistical Methodology Metadata (SDSM). It is being designed as an extension to the Cultural and Demographic Data Metadata (CDDM) draft standard of the Federal Geographic Data Committee (FGDC). Since statistical data has a geographic component, the FGDC standards are applicable to BOC data. In summer 1996, the SDSM will be submitted to the formal BOC standards development process, and is scheduled to be adopted in October.

The need for the SDSM became apparent from the results of early work to develop a prototype metadata repository. Work to build a metamodel for the repository revealed the importance of a standard set of descriptors for BOC data. The SDSM will contain all the information needed to describe survey designs, processing, analyses, and data sets for BOC surveys, and a metamodel will be designed from it.

The metamodel will be used to design a logically central repository of metadata for BOC survey data products. It will be an important (central) part of a unified data/metadata system.

In addition to the standard and the metamodel, a table of contents (TOC), or outline, of the contents of the SDSM is being built. The table of contents serves three main purposes:

- a model for displaying the contents of the SDSM and a vehicle for receiving comments;

- an outline of information which users can employ to locate or identify information they want to retrieve or supply; and

- a means to map the information contained in a repository to the information in another repository (as long as the other repository also has a table of contents).

The TOC has been installed on the WWW and will be available soon for Web users to make comments about its content.

The TOC outline is a metaphor for the information and subjects available to the users of a SIS. Developers of SIS's should build a table of contents as part of the user interface to their system.

The TOC can be used as a means for communication between systems. The metadata and metamodel for each system should be based on a metadata standard which can be represented in outline form. Because statistical metadata data elements are the same across survey programs, there should be a mapping linking the information between the systems using the tables of contents.

Data elements are the fundamental units of data an organization keeps and disseminates. Standardizing data elements promotes the understanding, sharing, and using of data.

Data elements and their associated metadata are an integral part of a SIS. Variable definitions permeate all SIS processes and the metamodel. They are used in all aspects of survey work. They are the lingua franca of a SIS.

Statistical agencies which administer many surveys define many variables. Variables such as income often have different definitions in different surveys. Users who wish to combine data sets based on variable names need to be aware of the differences in usage. Data administrators, survey designers, analysts, and researchers need to know which variables are the same and which are different. Data element standards will promote solutions to these problems.

There is an emerging data element standard under the aegis of ISO (the International Organization for Standards): ISO/IEC 11179 - Specification and Standardization of Data Elements. The basic focus of the standard is the requirements for maintaining information about an organization's data elements in a registry. The group that maintains the registry will be called the registration authority. A data element registry will be an integral part of a metadata repository.

It is very important for statistical agencies to implement standards such as ISO/IEC 11179. Sharing information with users is a process which will continue as long as there is a need for data.

\subsection{Architecture and principles}

An overview for the architecture of a SIS was discussed in chapter 2 . Here, the point is not a specific architecture, but that unification of SIS's is facilitated by common architectural features. These features can be illustrated by listing some principles for the design of a unified data/metadata system.

The following is a list of those principles:

- provide internet access;

- use WWW browsers for graphical user interface;

- use HTML, key word searches, and WAIS for document retrieval and information location;

-use metadata standards to design metadata repository models;

- create tables of contents (from metadata standards) for mapping information across systems;

-use tables of contents to provide information outline to users and analysts;

- provide common or standard software systems for data storage, retrieval, and analysis;

-employ data administration standards to standardize data elements.

This list may not be complete, but it represents the most important features a SIS should have. If all the SIS development projects of a statistical agency employ these principles, it will be much easier to bring the systems into a consistent, seamless, coherent, unified system. 


\section{Unified data/metadata system}

Here additional functionality and an architecture for a unified system are described.

\subsection{Additional functions}

There would be no purpose to building a unified system if there were no additional capabilities available. Here we give a list of the most important ones:

- build data sets from multiple sources;

- search for information across many program areas;

- compare survey designs across program areas;

- implement data element standards across program areas;

- lower respondent burden by identifying duplicate data collection.

This list is not inclusive, but is presented to give an idea of the power of a unified system.

\subsection{Architecture}

The central new piece to a unified system is the logically central metadata repository. Each SIS has a metadata component which has information describing its data sets. The central repository (We will leave off the "logically", but this repository will probably be distributed.) will have information about all the data in the statistical agency.

This will necessitate duplicating some information, but there are some advantages to that. Each SIS will be available to the survey designers in each program area. Designing and updating documentation and metadata is a process which requires some review and experiment. Each individual program area SIS will be where this work is done. After changes are accepted, the new metadata will be automatically mirrored to the central repository. It will then become available to everyone.

Because of the demand on the metadata and documentation in each SIS by survey designers and analysts, the central repository will be used by the SIS to show users the remainder of the information potential from its perspective. Users will be able to find data which is not in a particular program area SIS. This will decrease demand on any particular metadata repository: the central repository handling information requests from users who do not care or know about the sources of the data and the individual SIS repositories handling the requests from the users who desire a particular source.

Tables of contents for each metadata repository will be the communication interface between systems. The central repository duplicates information from each SIS and contains some additional information. Its metamodel is complete. The metamodels of each SIS can be considered a subset of the central metamodel. So, all communication can be filtered through the central system.
This scheme has several advantages. First, the number of communication interfaces is reduced. If there are $\mathbf{n}$ SIS's, then there are $\mathbf{n}$ interfaces, each with the central repository. Second, there are standards, such as CORBA, which address the routing of information in a communications architecture like this. It may be possible to build sophisticated routing requests to the individual survey data and documentation. Last, tags which identify individual metadata items in the repositories can easily be associated with entries in the tables of contents. The tags are defined in the SDSM standard. Thus, requests for information can be easily transferred across systems.

Only the metadata will be mirrored in the central repository. Each SIS has control over the survey data sets and documentation. The documentation will reside on individual file servers. The metadata itself will probably be small compared with the size of all the data and documents. Pointers, URL's (Uniform Resource Locators), to data sets and documents will be stored in the metadata repositories.

Figure 2 below shows a possible configuration for the architecture described above.

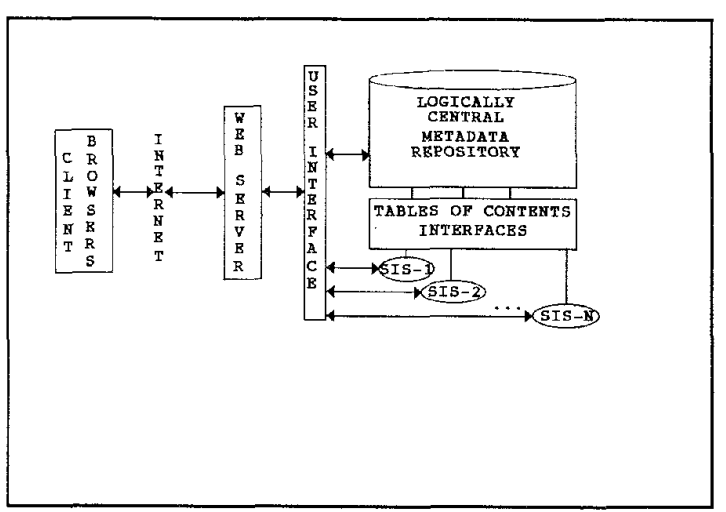

Figure 2. Unified System Architecture

\section{Conclusion}

This paper described principles used to guide the design of a unified data/metadata system, some important metadata standards, useful software tools, and a proposed architecture.

There are many data dissemination projects under development at the BOC at this time. Few of the designers and developers of these projects are talking to each other and coordinating their work. The authors and a few others are trying to bridge this communication gap. This paper represents the authors' views on where the coordination across projects could go. 


\section{Bibliography}

Almond, J. (1994), "Ideas for Information Types and Metadata Attributes", Center for High Performance Computing, Feb. 1994.

ANSI X3L8 - Data Representations (1996), "ISO/IEC 11179 Part 1 - Framework for the Specification and Standardization of Data Elements, Working Draft 7", February 1996.

Bretherton, F. (1994), "Reference Model for Metadata A Strawman", University of Wisconsin, draft, 1994.

Capps, C. (1995), "Overview of the Technical Architecture for FERRET", Census Bureau internal document, Demographic Surveys Division.

Census Bureau (1995), "FY 95 Internet Activities and Responsibilities", Census Bureau internal memorandum from Martha Farnsworth Riche, Director, January 16, 1995.

Census Bureau (1996), "Standard for Survey Design and Statistical Methodology Metadata" Draft Standard, Census Bureau internal document, in progress.

Census Bureau (1996), "Table of Contents for Survey Design and Statistical Methodology Metadata, Draft Standard", Census Bureau internal document, in progress.

FGDC (1994a), Federal Geographic Data Committee, "Content Standards for Digital Geospatial Metadata", June 8, 1994.

FGDC (1994b), Federal Geographic Data Committee Subcommittee on Cultural and Demographic Data, "Cultural and Demographic Data Metadata", DRAFT, September 15, 1994.

Gillman, D. W. and Appel, M. V. (1994), "Metadata Database Development at the Census Bureau", Presented at the UN/ECE METIS Working Group Meeting, Geneva Switzerland, November 22-25, 1994.

Internet Management Team (1994), "Internet Prototype Final Report", Bureau of the Census, October 1994.

Jackson, A. and Matchett, S. (1994), "Internet Joint Ventures", Census Bureau internal memorandum from
Valerie Gregg, DIAMOND Staff, July 13, 1994.

Lenz, H.-J. (1994), "The Conceptual Schema and External Schemata of Metadatabases", Proceedings of SSDBM-7, pp160-165, Charlottesville, VA, September 28-30, 1994.

Malmborg, E. and Sundgren, B. (1994), "Integration of Statistical Information Systems - Theory and Practice", Proceedings of SSDBM-7, pp80-89, Charlottesville, VA, September 28-30, 1994.

NIST (1989), National Institute for Standards and Technology, "Information Resource Dictionary System (IRDS)", Federal Information Processing Standard (FIPS) Publication 156, April 5, 1989.

Reinvention Lab of the Census Bureau (1994), "Integrated Processing System", Systems Planning Document, December 15, 1994.

Rosen, B. and Sundgren, B. (1991), "Documentation for Reuse of Microdata from the Surveys Carried Out by Statistics Sweden", Research and Development Statistics Sweden, June 28, 1991.

Sumpter, R. M. (1994), "Whitepaper on Data Management", Lawrence Livermore National Laboratory document, 1994.

Sundgren, B. (1991a), "Towards a Unified Data and Metadata System at the Australian Bureau of Statistics Final Report, December 2, 1991.

Sundgren, B. (1991b), "Statistical Metainformation and Metainformation Systems", R\&D Report Statistics Sweden, 1991:11.

Sundgren, B. (1992), "Organizing the Metainformation Systems of a Statistical Office", R\&D Report Statistics Sweden, 1992:10.

Sundgren, B. (1993), "Guidelines on the Design and Implementation of Statistical Metainformation Systems", R\&D Report Statistics Sweden, 1993:4.

Sundgren, B., Gillman, D. W., Appel, M. V., and LaPlant, W. P. (1996), "Towards a Unified Data and Metadata System at the Census Bureau", DRAFT Census Bureau Report, April 1995 